\title{
Improved atom equivalents method for converting density functional theory energies calculated on molecular mechanics structures to heats of formation
}

\author{
Pablo Duchowicz and Eduardo A. Castro* \\ CEQUINOR, Departamento de Química, Facultad de Ciencias Exactas, Universidad \\ Nacional de La Plata, C.C. 962, La Plata 1900, Argentina \\ E-mail: castro@dalton.quimica.unlp.edu.ar
}

(received 26 Jun 05; accepted 10 Dec 01; published on the web 18 Dec 01)

\begin{abstract}
We present an improved atom equivalents method for converting Density Functional Theory energies calculated on molecular mechanics structures to enthalpies of formation. The introduction of bond parameters to an original sent of atom parameters yields a sensible improvement of the enthalpy predictions. The comparison with other similar approaches shows that our present method is a rather better calculation scheme. Some further possible improvements are pointed out.
\end{abstract}

Keywords: Density functional theory energy, enthalpy of formation, atom equivalents method

\section{Introduction}

The requirements of the ever increasing development of pure and applied chemistry demands a reliable and as large as possible databank on Thermodynamic and Kinetic values, such as bond dissociation enthalpies, standard molar enthalpies of formation, energies of activation, etc. Such data are of paramount importance to the understanding of chemical problems and fruitful applications, such as energetics of the chemical bonds, structural properties and reactivity, chemical industry, biochemistry, environmental chemistry and many other equally important ${ }^{1}$.

Notwithstanding, there is a huge difference between the size of the experimental thermochemical databanks and the number of known molecules, and this gap increases 
steadily since it is not possible to measure the thermochemical properties for all the existing compounds and for the new one synthesized day after day. For the time being, the situation is such that thermochemical data on some classes of compounds, such as hydrocarbons and other aliphatic compounds, are well known and of quite reliable quality, but on other cases, like aromatic and heterocycle compounds, those data are scarce or even totally lacking. Hence, it is necessary to have credible and accurate enough methods for estimating the enthalpies of formation of different sort of compounds.

At present, there are several well-established theoretical schemes for the estimation of these properties, although each of them is suitable for a reduced set of molecules or/and they are questionable in some sense, so that it remains rather difficult to apply them routinely to arbitrary organic compounds.

In a recent contribution aiming to overcome the previously mentioned drawbacks, Rousseau and Mathieu have presented new atom equivalents to convert Density Functional Theory (DFT) energies calculated on molecular mechanics structures to formation enthalpies $\left(\Delta \mathrm{H}_{\mathrm{f}}^{\mathrm{o}}\right)^{2}$. The central purpose of the authors was to look for practical and widely applicable methods and more efficient procedures, and their results compare well with other similar schemes based on previous atom equivalents techniques. This study could demonstrate that cost-effective approaches to molecular formation enthalpies may be developed just combining DFT with an appropriate molecular mechanics force field.

The purpose of the present work is to take a step further on the method introducing bond parameters in order to take into account explicitly the neighborhood effects. This sort of correction has shown to be a suitable way to improve similar atom equivalents methods to compute $\Delta \mathrm{H}_{\mathrm{f}}^{\mathrm{o}} \cdot{ }^{3-6}$

\section{Method}

The well known idea of transferability has played a significant role in the whole area of physical chemistry. The very concepts of molecular size, molecular shape, and molecular structure are firmly settled in the modern chemistry around 200 years ago. ${ }^{7}$

The classical theory of the chemical compound structures conceived the molecule as a set of single interacting effective atoms. This well-known concept also implies the transferability and additivity of the molecular features connected with identical atoms and bonds. The principle of transferability has often been called for to analyse a wide variety of physical chemistry properties. ${ }^{8-14}$ Whenever a chemical bond is regarded to be independent of its location in some molecular structure, or the additivity of several parameters like bond moments, covalent radii or bond energies, is accepted, the validity 
of the principle of transferability is being assumed and made evident. Consequently, theoretical physical chemists are in search of suitable parameters that might consistently carry the essential and meaningful chemical information assignable from one molecule to another, without major changes. ${ }^{15}$

In this regard, quite different sets of atom and group equivalents have been developed which allow a direct calculation of heats of formation from semiempirical and ab initio total electronic energies. ${ }^{16-19}$ All these procedures are based upon the very concept of transferability and they give a reasonable agreement with the available experimental data.

Recently, Rousseau and Mathieu ${ }^{2}$ have introduced new atom equivalents to convert $\mathrm{BP} / \mathrm{DN} * * / / \mathrm{MMFF}$ energies into $\Delta \mathrm{H}_{\mathrm{f}}^{\mathrm{o}}$ and the results compare rather well with the results of previous similar schemes. The basic starting equation is where

$$
\Delta \mathrm{H}_{\mathrm{f}}^{\circ}=\mathrm{E}+\mathrm{ZPE}+\mathrm{TR}+\mathrm{ROT}+\mathrm{VIB}+\mathrm{kT}+\sum_{k} H_{k}{ }^{\circ}+\sum_{k} X_{k}^{\prime}
$$

$\mathrm{H}_{\mathrm{k}}^{\mathrm{o}}$ is the formation enthalpy of the $\mathrm{k}$ gaseous atoms

$\mathrm{X}_{\mathrm{k}}^{\prime}$ is the atom equivalent

$\mathrm{E}$ is the total molecular electronic energy at the equilibrium geometry

$\mathrm{ZPE}$ is the zero-point energy

TR is the translational thermal energy

ROT is the rotational thermal energy

VIB is the vibrational thermal energy

kT converts energy into enthalpy

$\mathrm{X}^{\prime}{ }_{\mathrm{k}}$ is obtained from a least-squares fit of suitable experimental data. Eq. (1) can be simplified at a rather large extent since the explicit inclusion of TR, ROT and kT contributions, using calculated data, does not improve significantly the results. Besides, the atomic formation enthalpies $\mathrm{H}_{\mathrm{k}}^{\mathrm{o}}$ are included within new empirical paramters $\mathrm{Y}_{\mathrm{k}}$ together with ZPE and VIB, so that it is a usual practice in this sort of approximation to apply the following approximation. ${ }^{23,30-34}$

$$
\Delta \mathrm{H}_{\mathrm{f}}^{\circ}=\mathrm{E}+\sum_{k} Y_{k}
$$

The distinctive feature of this procedure proposed by Rousseau and Mathieu stems from the fact that it relies on DFT energies E calculated on molecular mechanics 
structures. Although numerical estimations based on Eq. (2) gives sensible $\Delta \mathrm{H}_{\mathrm{o}_{\mathrm{f}}}$ values, the authors pointed out some possible future developments of atomic equivalents approaches. One of these consists on using different atomic equivalents according to the chemical environment of the atoms. However, this procedure should increase at a somewhat large extent the number of atomic parameters in order to take into account the great diversity of such environments. Consequently, a nearly equivalent method to consider the existing atoms in a network of specific chemical bonds is to extend Eq. (2) as follows where the index k-1 runs over all chemical bonds an $\mathrm{W}$ is a constant.

$$
\Delta \mathrm{H}_{\mathrm{f}}^{\circ}=\mathrm{E}+\sum_{k} Y_{k}+\sum_{k} X_{k-1}+\mathrm{W}
$$

On the basis of previous results for $Y_{k}$, we have determined $X_{k-1}$ contributions as correction terms. That is to say, resorting to the Rousseau and Mathieu's parameters, we have computed the new $\mathrm{X}_{\mathrm{k}-1}$ bond parameters fitting them against experimental $\Delta \mathrm{H}_{\mathrm{f}}^{\mathrm{o}}$ values through Eq. (3), employing previous $\mathrm{Y}_{\mathrm{k}}$ 's.

In order to judge consistently the relative merits of the proposed methodology, we have resort to the same molecular sets as that used previously by Rousseau and Mathieu, and then we compare our results with their values as well as with those reported by Habibollahzdeh et al/35/ and Rice et $\mathrm{al}^{36}$. These specific choices are due these two works are based on atomic equivalents determined from DFT to estimate $\Delta \mathrm{H}_{\mathrm{f}}^{\mathrm{o}}$.

\section{Results and Discussion}

The training set used to derive the bond parameters together with $\Delta \mathrm{H}_{\mathrm{f}}^{\mathrm{o}}$ calculated by Rousseau and Mathieu ${ }^{2}$ and our present approach are given in Table 1, while in Table 2 we display atomic and bond parameters. Evidently, the training panel comprises a quite representative set of molecules including hydrocarbons, alcohols, ketones, aldehydes, ethers, carboxylic acids, nitriles, and halogenated hydrocarbons.

The analysis of results shown in Table 1 allows one to verify the improvements of predictions of heats of formation when introducing bond parameters. In particular, rms and maximum absolute deviation are lower for the present approach.

In Table 3 we present our estimations of $\Delta \mathrm{H}_{\mathrm{f}}^{\mathrm{o}}$ for the prediction molecular panel together with other theoretical results for comparative purposes. Maximum deviations 
and rms errors are also included to facilitate the corresponding comparisons among the different models.

The analysis of results shows that bond parameters are indeed "correction terms" regarding atomic parameters (see relative magnitudes of these quantities in Table 2). Since dimethylfuroxan presents an oxygen atom linked to one aza nitrogen atom through a dative bond, it cannot be handled with the MMFF force field, hence the lack of calculated value of this molecule. Once again, the insertion of corrective bond terms in Eq. (2) to give Eq. (3) yields ameliorate results, since there is an improvement of rms and maximum absolute deviations. The comparison of the results arising from the different methods enables one to verify that the introduction of bond parameters into the approximation of atom equivalents for converting DFT energies calculated on molecular mechanics structures to formation enthalpies yields a rather satisfactory theoretical scheme. Since experimental uncertainties in the determination of enthalpies of formation are around $10 \mathrm{~kJ} / \mathrm{mol}$, the average deviations are rather sensible. It is also interesting to compare our present approach with Habibollahzadeh et al's method, since this last one has been particularly designed to compute thermochemical data. Results of Table 3 show that maximum absolute deviations are similar, while our approach gives a lower root mean square deviation.

Table 1. Training set used to derive the bond parameters listed in Table 2

\begin{tabular}{lcccc}
\hline \multicolumn{1}{c}{ Molecule } & $\Delta \mathrm{H}_{\mathrm{f}}^{\circ}\left(\mathrm{BP} / \mathrm{DN}^{* *}\right)^{2 a}$ & $\Delta \mathrm{H}_{\mathrm{f}}^{\mathrm{o}}$ (this work) & $\Delta_{1}^{2 b}$ & $\begin{array}{c}\Delta_{2}(\text { this } \\
\text { work })^{b}\end{array}$ \\
\hline 2,2,3,3-Tetrafluoro-1-propanol & -1061.3 & -1064.6 & 7.3 & 4.0 \\
Hexafluorobenzene & -956.0 & -956.7 & -2.5 & -3.2 \\
Tetrafluoromethane & -933.2 & -939.1 & 18.6 & 12.69 \\
Pentafluorobenzene & -806.0 & -807.5 & -4.0 & -5.5 \\
Chlorotrifluoromethane & -707.9 & -711.3 & 7.9 & 4.5 \\
Trifluoromethane & -697.1 & -699.1 & 4.0 & 2.0 \\
1,2,4,5-Tetrafluorobenzene & -646.8 & -648.6 & -11.0 & -12.8 \\
3-Methylbutanoic acid & -510.0 & -509.4 & 1.0 & 1.6 \\
\hline
\end{tabular}


Table 1. Continued

\begin{tabular}{|c|c|c|c|c|}
\hline Trifluoroacetonitrile & -497.9 & -499.1 & 7.2 & 6.0 \\
\hline Butanoic acid & -475.9 & -473.8 & -10.9 & -8.8 \\
\hline Chlorodifluoromethane & -482.0 & -482.1 & -4.0 & -4.1 \\
\hline 2,3-Butanediol & -482.3 & -480.8 & 22.4 & 23.9 \\
\hline Difluoromethane & -450.7 & -448.3 & -3.2 & -0.8 \\
\hline 1,2,4-Benzenetriol & -444.0 & -444.1 & -8.4 & -8.5 \\
\hline Propyl formate & -462.7 & -459.8 & 33.1 & 36.0 \\
\hline Methyl acetate & -410.0 & -404.5 & -15.1 & -9.6 \\
\hline 1,4-Dioxane & -315.3 & -314.9 & -18.1 & -17.7 \\
\hline Hexanol & -315.9 & -313.5 & -10.2 & -7.8 \\
\hline Cyclohexanol & -286.2 & -292.7 & -8.1 & -14.6 \\
\hline 2-Propanol & -272.6 & -271.6 & -3.4 & -2.4 \\
\hline Acetamide & -238.3 & -232.6 & -15.8 & -10.1 \\
\hline Urea & -235.5 & -229.7 & -13.6 & -7.8 \\
\hline Ethanol & -243.8 & -238.8 & 3.1 & 8.1 \\
\hline Cyclopentanol & -242.5 & -247.0 & 2.0 & -2.5 \\
\hline Fluoromethane & -234.3 & -229.0 & -1.7 & 3.6 \\
\hline Acetone & -217.1 & -210.1 & -6.1 & 0.9 \\
\hline N,N-Dimethylformamide & -192.4 & -184.3 & -22.3 & -14.2 \\
\hline Formamide & -186.0 & -173.3 & -23.2 & -10.5 \\
\hline Propanal & -185.6 & -176.9 & -10.0 & -1.3 \\
\hline 2,2,3-Trimethylbutane & -204.5 & -2004.6 & 16.0 & 15.9 \\
\hline 2,2-Dimethylbutane & -185.6 & -185.0 & 9.9 & 10.5 \\
\hline Ethanal & -166.2 & -156.7 & -5.6 & 3.9 \\
\hline 2-Methyl-2-nitropropane & -177.1 & -179.3 & 5.4 & 3.2 \\
\hline 2,3-Dimethylbutane & -177.8 & -176.8 & 6.2 & 7.2 \\
\hline
\end{tabular}


Table 1. Continued

\begin{tabular}{|c|c|c|c|c|}
\hline Neopentane & -167.9 & -166.7 & 0.6 & 1.8 \\
\hline Nitrobutane & -143.9 & -144.3 & -15.9 & -16.3 \\
\hline 2-Methylbutane & -153.7 & -151.7 & -3.7 & -1.7 \\
\hline 1-Fluoro-4-methylbenzene & -147.5 & -148.8 & 0.7 & -0.6 \\
\hline Isobutane & -134.2 & -131.5 & -4.2 & -1.5 \\
\hline Ethoxyethene & -140.8 & -137.7 & 3.0 & 6.1 \\
\hline n-Butane & -127.1 & -124.0 & -7.2 & -4.1 \\
\hline Cyclohexane & -123.4 & -130.0 & -7.7 & -14.2 \\
\hline Cyclooctane & -124.4 & -130.3 & 2.6 & -3.3 \\
\hline Cycloheptane & -118.1 & -124.0 & -2.3 & -8.2 \\
\hline Chloroethane & -124.5 & -122.7 & 4.3 & 6.1 \\
\hline Nitroethane & -102.3 & -101.1 & -14.4 & -13.2 \\
\hline Propane & -104.7 & -100.7 & -9.3 & -5.3 \\
\hline Formaldehyde & -115.9 & -104.0 & 8.1 & 20.0 \\
\hline Chloroform & -103.2 & -102.2 & -1.3 & -0.3 \\
\hline Butylamine & -91.9 & -86.6 & -5.3 & 0.0 \\
\hline Ethane & -83.7 & -78.9 & -7.3 & -2.5 \\
\hline Methyl chloride & -83.7 & -80.0 & -3.9 & -0.2 \\
\hline Hydrogen chloride & -92.3 & -97.3 & 6.9 & 1.9 \\
\hline Cyclopentane & -76.4 & -80.7 & -5.6 & -9.9 \\
\hline Phenol & -96.4 & -105.0 & 14.6 & 6.0 \\
\hline Methane & -74.5 & -69.5 & -0.9 & 4.1 \\
\hline $\begin{array}{l}\text { 2-Methyl-1,2- } \\
\text { propanediamine }\end{array}$ & -90.3 & -84.7 & 18.1 & 23.7 \\
\hline Dinitromethane & -58.9 & -58.2 & 12.4 & 13.1 \\
\hline Methylamine & -22.5 & -14.4 & -2.2 & 5.9 \\
\hline Dimethylamine & -19.5 & -11.2 & -5.0 & 3.3 \\
\hline
\end{tabular}


Table 1. Continued

\begin{tabular}{|c|c|c|c|c|}
\hline Tetrachloroethylene & -12.4 & -15.2 & 1.4 & -1.4 \\
\hline 1,2,3-Trimethylbenzene & -9.5 & -11.6 & 2.3 & 0.2 \\
\hline p-Xylene & 18.0 & 17.1 & -0.9 & -1.8 \\
\hline 2-Methyl-propanenitrile & 23.4 & 24.4 & -5.0 & -4.0 \\
\hline Butanenitrile & 33.6 & 35.1 & -15.0 & -13.5 \\
\hline 1,2-Dimethylbenzene & 19.1 & 18.3 & 0.1 & -0.7 \\
\hline Cyclobutane & 28.4 & 29.5 & -8.3 & -7.2 \\
\hline 1,3-Dimethylbenzene & 17.3 & 16.4 & 3.6 & 2.7 \\
\hline Acetaldimine & 24.0 & -0.6 & 9.2 & -15.4 \\
\hline Propanenitrile & 51.7 & 53.9 & -9.7 & -7.5 \\
\hline Methylbenzene & 50.4 & 50.9 & -2.0 & -1.5 \\
\hline Chlorobenzene & 51.8 & 51.3 & -1.2 & -1.7 \\
\hline Ethanenitrile & 74.0 & 77.0 & -12.0 & -9.0 \\
\hline Ethylenediamine & 84.1 & 90.2 & -10.8 & -4.7 \\
\hline Cyclopropylamine & 77.0 & 80.7 & -1.3 & 2.4 \\
\hline Benzene & 82.9 & 84.9 & -3.0 & -1.0 \\
\hline N-Methylmethanimine & 44.0 & 20.2 & 39.2 & 15.4 \\
\hline 4-Methylpyridine & 103.8 & 115.8 & -12.0 & 0.0 \\
\hline 1H-Imidazole & 139.3 & 156.8 & -25.7 & -8.2 \\
\hline Hydrogen cyanide & 135.1 & 141.1 & -4.2 & 1.8 \\
\hline 1H-Pyrazole & 179.4 & 209.7 & -30.3 & 0.0 \\
\hline Butyne & 165.2 & 164.8 & -0.1 & -0.5 \\
\hline Dimethyl.diazene & 148.6 & 122.5 & 26.1 & 0.0 \\
\hline Propyne & 184.9 & 185.3 & 0.1 & 0.5 \\
\hline 4-Pyridinecarbonitrile & 283.5 & 295.0 & -19.5 & -8.0 \\
\hline Acridine & 273.9 & 277.3 & 12.8 & 16.2 \\
\hline
\end{tabular}


Table 1. Continued

\begin{tabular}{lcccc}
\hline $\begin{array}{l}\text { Bicyclo[1.1.0]butane } \\
\text { 1 carbonitrile }\end{array}$ & 304.5 & 300.1 & 38.6 & 34.2 \\
Rms & - & - & 12.6 & 10.1 \\
Maximum absolute deviation & - & - & 39.2 & 36.0 \\
\hline
\end{tabular}

${ }^{a}$ unit $\mathrm{kJ} / \mathrm{mol}$.

${ }^{b}$ error with respect to the experimental data. ${ }^{37}$

Table 2. Atom and bond equivalents taken from Ref. 2 and derived in this work, respectively $(\mathrm{kJ} / \mathrm{mol})$

\begin{tabular}{|c|c|c|c|}
\hline Atom and bond types & Coordination & $Y_{k}$ & $X_{k-1}$ \\
\hline $\mathrm{H}$ & 1 & 1557.6 & - \\
\hline $\mathrm{C}$ & 4 & $100,114.9$ & - \\
\hline $\mathrm{C}$ & 3 & $100,119.5$ & - \\
\hline $\mathrm{C}$ & 2 & $100,114.4$ & - \\
\hline $\mathrm{N}$ & 3 & $143,859.4$ & - \\
\hline $\mathrm{N}$ (nitro) & 3 & $143,908.2$ & - \\
\hline $\mathrm{N}$ & 2 & $143,856.5$ & - \\
\hline $\mathrm{N}$ & 1 & $143,846.6$ & - \\
\hline $\mathrm{O}$ & 2 & $197,426.1$ & - \\
\hline $\mathrm{O}$ & 1 & $197,427.9$ & - \\
\hline $\mathrm{F}$ & 1 & $262,042.9$ & - \\
\hline $\mathrm{Cl}$ & 1 & $1,208,399.3$ & - \\
\hline $\mathrm{C}-\mathrm{C}$ & - & - & -1.2215 \\
\hline $\mathrm{C}-\mathrm{H}$ & - & - & 0.6528 \\
\hline $\mathrm{C}-\mathrm{F}$ & - & - & 2.8234 \\
\hline $\mathrm{C}-\mathrm{O}$ & - & - & 0.4742 \\
\hline $\mathrm{O}-\mathrm{H}$ & - & - & 2.3221 \\
\hline $\mathrm{C}-\mathrm{C}_{\text {arom }}$ & - & - & -0.6818 \\
\hline $\mathrm{C}-\mathrm{Cl}$ & - & - & 0.4643 \\
\hline $\mathrm{C}=\mathrm{O}$ & - & - & 3.4990 \\
\hline $\mathrm{C} \equiv \mathrm{N}$ & - & - & -0.5397 \\
\hline $\mathrm{N}-\mathrm{H}$ & - & - & 0.8837 \\
\hline
\end{tabular}


Table 2. Continued

\begin{tabular}{cccc}
\hline $\mathrm{C}-\mathrm{N}$ & - & - & -1.0563 \\
$\mathrm{~N}-\mathrm{O}$ & - & - & 0.5485 \\
$\mathrm{~N}=\mathrm{O}$ & - & - & 0.5485 \\
$\mathrm{C}=\mathrm{C}$ & - & - & -1.9198 \\
$\mathrm{C}-\mathrm{N}_{\text {arom }}$ & - & - & 0.6690 \\
$\mathrm{~N}-\mathrm{N}_{\text {arom }}$ & - & - & 2.5135 \\
$\mathrm{C} \equiv \mathrm{C}$ & - & - & -3.2865 \\
$\mathrm{C}=\mathrm{N}$ & - & - & -8.0259 \\
$\mathrm{~N}=\mathrm{N}$ & - & - & -9.5493 \\
\hline
\end{tabular}

Constant term in Eq. (3) (W) equal to -0.4453 .

Table 3. Enthalpies of formation $(\mathrm{kJ} / \mathrm{mol})$ for several compounds

\begin{tabular}{|c|c|c|c|c|c|c|}
\hline Molecule & Exp. ${ }^{38}$ & PIMM $^{2}$ & $\mathrm{P} 1^{2}$ & $\mathrm{P} 2^{2}$ & $\mathrm{HGCMP}^{35}$ & This work \\
\hline \multirow[t]{2}{*}{ Chlorodifluoromethane } & -482.0 & -438.6 & - & - & -461.7 & -486.2 \\
\hline & & & 472.6 & 485.9 & & \\
\hline \multirow[t]{2}{*}{ Methyl acetate } & -411.5 & -412.9 & - & - & -405.6 & -422.4 \\
\hline & & & 423.3 & 426.9 & & \\
\hline \multirow[t]{2}{*}{ Ethylene glycol } & -387.1 & -411.1 & - & - & -357.9 & -380.0 \\
\hline & & & 374.0 & 386.1 & & \\
\hline \multirow[t]{2}{*}{ Formic acid } & -378.6 & -371.8 & - & - & -352.8 & -387.1 \\
\hline & & & 385.7 & 392.7 & & \\
\hline \multirow[t]{2}{*}{ 1,4-Dioxane } & -315.5 & -307.2 & - & - & -332.8 & -299.6 \\
\hline & & & 296.5 & 300.8 & & \\
\hline \multirow[t]{2}{*}{ 1-Fluoropropane } & -285.6 & -285.1 & - & - & -272.8 & -293.6 \\
\hline & & & 293.1 & 296.2 & & \\
\hline \multirow[t]{2}{*}{ Diethyl ether } & -251.8 & -253.0 & - & - & -265.1 & -262.7 \\
\hline & & & 268.7 & 267.2 & & \\
\hline \multirow[t]{2}{*}{ Urea } & -245.6 & -211.4 & - & - & -211.3 & -236.2 \\
\hline & & & 234.9 & 241.6 & & \\
\hline \multirow[t]{2}{*}{ Water } & -241.6 & -234.3 & - & - & -197.8 & -229.1 \\
\hline & & & 227.9 & 233.8 & & \\
\hline \multirow[t]{2}{*}{ Acetamide } & -238.1 & -214.0 & - & - & -224.5 & -246.3 \\
\hline & & & 248.3 & 251.3 & & \\
\hline
\end{tabular}


Table 3. Continued

\begin{tabular}{|c|c|c|c|c|c|c|}
\hline Ethanol & -235.0 & -242.1 & -233.3 & -240.7 & -219.6 & -235.6 \\
\hline Acetone & -217.1 & -209.7 & -224.1 & -223.2 & -196.3 & -216.5 \\
\hline Methanol & -201.3 & -219.6 & -197.9 & -204.5 & -183.6 & -197.7 \\
\hline Dimethylether & -183.9 & -208.0 & -189.9 & -195.3 & -184.7 & -187.5 \\
\hline Neopentane & -168.0 & -175.0 & -169.0 & -167.3 & -154.5 & -166.0 \\
\hline n-Butane & -125.5 & -127.9 & -132.6 & -134.3 & -127.3 & -131.6 \\
\hline Methyl nitrate & -122.1 & -189.9 & -154.0 & -158.2 & -163.9 & -151.7 \\
\hline Chloroethane & -112.0 & -100.8 & -108.1 & -120.3 & -117.9 & -118.3 \\
\hline Formaldehyde & -108.5 & -120.8 & -108.8 & -107.8 & -79.8 & -95.5 \\
\hline Propane & -104.6 & -106.2 & -113.4 & -114.0 & -105.8 & -110.5 \\
\hline Nitroethane & -102.2 & -121.8 & -115.5 & -116.7 & -84.1 & -116.3 \\
\hline Ethane & -83.6 & -85.6 & -91.4 & -90.9 & -84.7 & -86.5 \\
\hline Nitromethane & -74.4 & -97.2 & -87.3 & -86.9 & -46.8 & -85.2 \\
\hline Methane & -74.4 & -65.3 & -78.8 & -75.4 & -70.6 & -70.5 \\
\hline Methyl nitrite & -66.5 & -81.6 & -30.0 & -32.2 & -81.0 & -21.1 \\
\hline Ammonia & -46.0 & -9.4 & -41.8 & -42.7 & -22.9 & -32.1 \\
\hline Benzaldehyde & -36.7 & -45.1 & -40.7 & -45.3 & -40.6 & -40.9 \\
\hline Furan & -34.9 & -61.7 & -26.0 & -42.5 & -54.2 & -44.3 \\
\hline Methylamine & -23.0 & -23.5 & -22.9 & -24.7 & -14.3 & -16.8 \\
\hline Isobutene & -16.9 & -2.7 & -14.8 & -15.9 & -11.0 & -15.0 \\
\hline Oxazole & -15.5 & -40.6 & -11.7 & -29.7 & -16.7 & -22.3 \\
\hline Dimethylnitramine & -4.6 & 30.1 & -26.7 & -26.3 & -11.2 & -17.5 \\
\hline Propene & 20.0 & 28.1 & 22.2 & 20.5 & 26.6 & 23.0 \\
\hline Vinyl chloride & 37.2 & 20.8 & 36.4 & 22.3 & 14.3 & 23.8 \\
\hline Nitroethylene & 37.6 & 2.0 & 45.0 & 41.0 & 59.4 & 41.7 \\
\hline $\begin{array}{l}\text { 4,5-dihydro-3- } \\
\text { nitroisoxazole }\end{array}$ & 39.0 & -34.8 & 28.0 & 53.1 & 50.0 & 25.7 \\
\hline Chlorobenzene & 51.8 & 52.4 & 64.1 & 50.4 & 39.3 & 49.8 \\
\hline $\begin{array}{l}\text { Triamino- } \\
\text { trinitrobenzene }\end{array}$ & 51.8 & -34.8 & 101.7 & 102.0 & 75.0 & 98.1 \\
\hline Ethylene & 52.3 & 59.5 & 59.1 & 57.7 & 65.3 & 61.9 \\
\hline Cyclopropane & 53.1 & 50.3 & 29.0 & 42.5 & 50.9 & 44.0 \\
\hline 1,3-Dinitrobenzene & 53.9 & 24.9 & 67.2 & 65.6 & 71.4 & 61.5 \\
\hline 1,4-Dinitropiperazine & 58.1 & 155.0 & 29.3 & 35.9 & 50.6 & 39.1 \\
\hline
\end{tabular}


Table 3. Continued

\begin{tabular}{|c|c|c|c|c|c|c|}
\hline 4-Nitroaniline & 58.9 & 34.6 & 87.3 & 83.6 & 54.0 & 83.3 \\
\hline 1,3,5-Trinitrobenzene & 62.3 & 46.5 & 81.1 & 80.0 & 76.2 & 74.1 \\
\hline Acetonitrile & 64.4 & 87.9 & 81.6 & 62.0 & 64.2 & 83.0 \\
\hline Nitrobenzene & 67.3 & 37.1 & 66.1 & 63.5 & 68.1 & 61.8 \\
\hline Isoxazole & 78.6 & 104.4 & 69.9 & 55.4 & 64.7 & 61.9 \\
\hline Benzene & 82.3 & 82.5 & 83.6 & 79.9 & 79.2 & 81.7 \\
\hline Aniline & 86.9 & 90.2 & 85.5 & 80.7 & 77.9 & 83.0 \\
\hline Methylhydrazine & 94.5 & 91.7 & 85.5 & 85.3 & 87.6 & 103.0 \\
\hline Dimethylfuroxan & 102.2 & 123.9 & - & - & 68.6 & - \\
\hline Dimethylfurazan & 107.2 & 160.4 & 80.8 & 76.0 & 82.6 & 87.2 \\
\hline Pyrrole & 108.2 & 74.4 & 104.2 & 100.3 & 101.4 & 115.6 \\
\hline 1,3-Butadiene & 109.9 & 110.4 & 122.2 & 116.4 & 118.9 & 116.2 \\
\hline Pyridine & 140.3 & 153.9 & 131.5 & 128.1 & 131.2 & 141.1 \\
\hline Indole & 156.3 & 169.5 & 158.2 & 154.7 & 139.1 & 164.2 \\
\hline Propyne & 184.7 & 185.1 & 184.3 & 185.0 & 178.2 & 185.4 \\
\hline Allene & 190.3 & - & 177.7 & 183.0 & 188.4 & 186.1 \\
\hline RDX & 191.4 & 240.7 & 178.7 & 185.1 & 167.6 & 197.1 \\
\hline Pyrimidine & 195.7 & 203.5 & 174.1 & 171.1 & 196.9 & 195.1 \\
\hline Pyrazine & 195.9 & 212.1 & 188.3 & 186.1 & 212.1 & 210.9 \\
\hline Acetylene & 228.0 & 227.1 & 140.0 & 240.1 & 234.5 & 243.2 \\
\hline Pyridazine & 278.1 & 284.3 & 258.7 & 258.4 & 282.2 & 278.7 \\
\hline Tetrazole & 334.2 & 359.7 & 290.6 & 292.5 & 332.1 & 318.8 \\
\hline rms absolute deviation & - & 28.7 & 15.7 & 15.5 & 17.1 & 13.6 \\
\hline $\begin{array}{l}\text { Maximum absolute } \\
\text { deviation }\end{array}$ & - & 96.9 & 59.6 & 64.8 & 43.8 & 46.3 \\
\hline
\end{tabular}

\section{Conclusions}

Following a similar basic perspective as that stated in Rousseau and Mathieu's paper ${ }^{2}$, we do not intend here to achieve for an extreme chemical accuracy but to look for a more efficient and simple way to estimate a fundamental thermodynamical quantity. This work aims to introduce an improvement into a recent method based on atom equivalents for converting DFT energies calculated on molecular mechanics structures to heats of 
formation. The improvement consists in inserting bond parameters in order to take into account the chemical environment of each atom within the molecule. This change does not introduce any further extra effort in the computational procedure so that the simplicity of the original approach ${ }^{2}$ is kept. This sort of amelioration is not new and some previous applications have shown the convenience of inserting it within the context of similar calculation schemes ${ }^{3-6}$. The basic idea behind the methodology is consistent with the variety of atom types involved in force field definitions and present results seem to suggest a suitable way to define the optimal combination of force field, electronic structure model, and the set of atomic equivalents and bond parameters.

Regarding some possible future improvements of the method described here, perhaps it should be advisable to define atom and bond parameters for rather more specific sets of molecules. In fact, although it seems the procedure leads to a "parameters nightmare", it should be more sensible since it is nearly impossible to attain a satisfactory enough agreement between experimental and theoretical data when the fitting set is composed of quite different molecules. It must be taken into account that this sort of methodology is a semiempirical one, where results arising from first principle methods and empirical molecular mechanics procedures are used together with arbitrary fitting equations and experimental data.

At present, research along this line is under development in our laboratory and results will be presented elsewhere in the forthcoming future.

\section{References and Notes}

1. Ribeiro da Silva, M. A. V. Pure Appl. Chem. 1999, 71, 71257.

2. Rousseau, E.; D. Mathieu, J. Comp. Chem 2000, 21, 1.

3. Duchowicz, P.; Castro, E. A. J. Kor. Chem. Soc. 1999, 43(6), 621.

4. Cioslowski, J.; Liu, G.; Piskorz, P. J. Phys. Chem. A 1998, 102, 9890.

5. Becerra, R.; Walsh, R. “Thermochemistry", In The Chemistry of Organic Silicon Compounds, Rappoport, Z.; Apeloig, Y. Eds; Wiley: New York, 1998.

6. Cox, J. D.; Pilcher, G. "Thermochemistry of Organic and Organometallic Compounds”, Academic Press: London-New York, 1970.

7. Dalton, J. “A New System of Chemical Philosophy”, 1806, reprinted in "Foundation of the Molecular Theory”, Alembic Club: Edinburgh, 1928.

8. Gineityte, V.; Shatkvoskaya, D. Int. J. Quantum Chem. 1991, 39, 11.

9. Kapuy, E.; Szalay, P. G.; Daudel, R.; Bogar, F.; Bartha, F.; Kozmutza, C. J. Mol. 
Struct. THEOCHEM 1991, 226, 351.

10. Price, S. L.; Faerman, C. H.; Murray, C. W. J. Comp.Chem. 1991, 12, 1187.

11. Barbiric, D. A.; Castro, E. A.; Fernández, F. M. Rev. Bol. Quim. 1991, 10, 5.

12. Castro, E. A. Fernández, F. M. Rev. Roum. Chim. 1989, 25, 455.

13. Goedecker, S.; Maschke, K. Phys. Rev. A. 1992, 45, 88.

14. Degand, G.; Leroy, G.; Peeters, D. Theor. Chim. Acta. 1973, 30, 243.

15. Castro, E. A. Comp. Chem. 1997, 21, 305.

16. Hehre, W. H.; Radom, L.; Schleyer, P. V. R.; Pople, J. A. Ab Initio Molecular Orbital Theory, Wiley: New York, 1984.

17. Hehre, W. J.; Ditchfield, R.; Radom, L.; Pople, J. A. J. Am. Chem. Soc. 1970, 92, 4796.

18. Schulman, J. M.; Disch, R. L. J. Am. Chem. Soc. 1984, 104, 1202.

19. Van Wazer, J. R.; Kell, V.; Hess, B. A.; Ewig, C. S. J. Phys. Chem. 1990, 94, 5694.

20. Castro, E. A. J. Mol. Struct. THEOCHEM. 1984, 304, 93.

21. Sana, M.; Nguyen, M. T. Chem. Phys. Lett. 1992, 196, 390.

22. Dewar, M. J. S.; Zoebisch, E. G.; Healy, E. F. Stewart, J. J. P. J. Am. Chem. Soc. 1985, 107, 39092.

23. Ibrahim, M. B. Schleyer, P. V. R. J. Comp. Chem. 1985, 6, 157.

24. Yala, Z. J. Mol. Struct. THEOCHEM. 1990, 207, 217.

25. Engelke, R. J. Am. Chem. Soc. 1993, 115, 2961.

26. Laurencelle, N.; Pacey, P. D. J. Am. Chem. Soc. 1993, 115, 625.

27. Benson, S. W. Computational Thermochemistry, ACS Symposium Series 677, American Chemical Society: Washington, DC, 1998.

28. Golan Mostafa, A. T.; Eakman, J. M.; Yarbro, S. L. Ind. Eng. Chem. Res. 1995, 34, 4577.

29. Donalski, E. S.; Hearing, E. D. J. Phys. Chem. Ref. Data 1993, 22, 805.

30. Smith, A.E. Lindner, H. J. Comp. Zid Mol. Des. 1991, 5, 231.

31. Kroeker, M.; Lindner, J. J. Mol. Mod. 1996, 2, 376.

32. Wiberg, K. B. J. Comp. Chem. 1984, 5, 197.

33. Wiberg, K. B. J. Org. Chem.

34. Mole, S. J.; Zhou, X.; Liu, R. J. Phys. Chem. 1996, 100, 14665.

35. Habibollahzadeh, D.; Grice, M. E.; Concha, M. C.; Murray, J. S.; Politzer, P. J. Comp. Chem. 1995,16, 654.

36. Rice, B. M.; Pai, S. V.; Hare, J. Combust. Flame 1999, 118, 445.

37. Lide, D. R. Ed., CRC Handbook of Chemistry and Physics, CRC Press: Boca Raton: FL, 1996, $77^{\text {th }}$ Edn. 
38. Mallard, W. G.; Linstrom, P. J. Eds; NIST Chemistry Webbook, NIST Standard Reference Database Number 69, November 1998, NIST, Gaithersburg, MD. 